\title{
La Revista SIAP como mecanismo para la consolidación del saber experto en América Latina.
}

\section{SIAP Journal as a Mechanism for the Consolidation of Expert Knowledge in Latin America}

Dossier: Cultura del espacio y cultura política en la Argentina 1966-2001

AlejandRa MonTI*

Centro Universitario Rosario de Investigaciones Urbanas y Regionales.

Facultad de Arquitectura, Planeamiento y Diseño. Universidad Nacional de Rosario/

CONICET

montialejandra@gmail.com

* Arquitecta (UNR). Magíster en Historia y Cultura de la Arquitectura y la Ciudad (UTDT) y Doctora en Arquitectura (UNR). Becaria Posdoctoral del CONICET. Docente del Área Teoría y Técnica Urbanística (FAPyD). Participa en proyectos de investigación en el Área de Historia de la Arquitectura y la Ciudad (UNR). 


\section{Resumen:}

En el año 1966 la Sociedad Interamericana de Planificación (SIAP) inicia su programa editorial denominado Ediciones SIAP. La Revista SIAP se constituye como uno de los ejes del mismo, convirtiéndose en el órgano de difusión de la sociedad. Esta condición permite pensar a la revista como el espacio de afirmación del monopolio experto sobre el discurso de la planificación regional y urbana, en un momento particular del giro teórico que paulatinamente va abandonando el discurso desarrollista por el dependentista. Centrar la indagación en el primer período de la revista (1967-1970) permite analizar los intentos por estabilizar y delimitar el marco teórico-metodológico de la planificación y el rol de los expertos en el marco de los procesos de transformación de la disciplina en la región.

Palabras Claves: Revista SIAP; Expertos; Planificación regional y urbana

\section{Abstracts:}

In 1966, the Inter-American Planning Society (SIAP) begins its editorial program called SIAP Editions. SIAP Journal becomes one of its lines, turning into the organ of diffusion of the society. This condition allows us to consider the journal as an assertion of the expertise monopoly about urban and regional planning discourse, in a particular moment of the theoretical turn that gradually abandons the development discourse for the dependency theory. Focusing the investigation in the first period of the journal (1967-1970) allows us to analyze the attempts to stabilize and define Planning theoretical and methodological framework and the role of the experts in the discipline transformation processes in the region.

Keywords: SIAP Journal; Expert; Urban and regional planning 
En los últimos años es posible observar un renovado interés en la disciplina urbana y territorial por comprender las condiciones que llevaron a la consolidación de los postulados de la planificación para el desarrollo en América Latina (Almandoz, 2006, 2010; Gorelik, 2014), en un período caracterizado por la reconfiguración de las fuerzas políticas, económicas y culturales del mundo, que consolidan el poder de los Estados Unidos en los años posteriores a la Segunda Guerra Mundial.

La renovación de las relaciones entre el país del norte y sus vecinos continentales, iniciada a partir de la firma de la Alianza para el Progreso en 1961, sumado a la emergencia de una serie de instituciones y centros que tienen a Latinoamérica como eje del debate, redefinen los modos y mecanismos de expansión y delimitación de la renovada disciplina. La cual asume un perfil regional a partir de la convicción de una pasado compartido y un presente común que debe ser transformado bajo el paradigma reformista-desarrollista imperante en el período.

La presencia de los Estados Unidos en la región adquiere diferentes formas y mecanismos. Por un lado, se presenta la actividad filantrópica de empresas privadas (Fundación Rockefeller, Fundación Ford) que operan en la diagramación de las agendas políticas y económicas de los países latinoamericanos. En segundo término, se impulsa la creación de instituciones panamericanas que cuentan con la asistencia financiera y técnica de organismos supranacionales. En último lugar, se ubica la ampliación de las formas de "contacto" estableciendo relaciones culturales de larga duración a partir del intercambio de estudiantes y docentes, que tiene a los Estados Unidos como destino de formación de posgrado para los profesionales latinoamericanos.

En este escenario transformado, una mirada sobre la Revista de la Sociedad Interamericana de Planificación (SIAP) nos permite indagar en las estrategias empleadas para la construcción y consolidación de un espacio de afirmación del monopolio experto sobre el discurso de la planificación regional y urbana en clave desarrollista en América Latina. Esta lectura, permite conocer sobre los espacios de producción de las ideas de la planificación y los diferentes actores que van a ir delineando el perfil de la publicación desde un posicionamiento inicial de cuño desarrollista y su paulatina transformación a posiciones más críticas vinculadas a la teoría de la dependencia.

En esta línea, en primer lugar se presentan las condiciones que permiten identificar a la Sociedad como un nuevo espacio de institucionalización de la disciplina en América Latina revisitada en clave desarrollista. En segundo término, nos interesa revisar el proyecto editorial propuesto por SIAP identificando los diferentes programas que se desarrollaron de manera simultánea a partir de su lanzamiento en el año 1966. Por último, centramos la indagación en los números Revista SIAP publicados durante el primer período de publicación, con el fin de identificar los diferentes temas abordados y su variación en el tiempo, sumado al reconocimiento de los autores y sus respectivas trayectorias, que permiten examinar los vínculos entre las ideas y los diferentes espacios de producción del ideal planificador latinoamericano.

\section{Mecanismos de transformación}

Sarfatti Larson (1988) desarrolla el concepto de estrategia profesional para dar cuenta del proceso histórico por el cual un determinado grupo de profesionales logra monopolizar un segmento específico del mercado de trabajo y consiguen reconocer su dominio con la ayuda del Estado. La autora identifica que en la operación del "aislamiento social" subyace la monopolización de un mercado de trabajo y la definición de un saber específico que se presenta diferenciado de otros campos del conocimiento.

Sostenemos que con la creación de SIAP en el año 1956, se introduce un nuevo proceso de institucionalización y delimitación de la disciplina urbana en el marco de un contexto más amplio de las transformaciones de las Ciencias Sociales en el continente.

Si bien desde 1920 ya existían una serie de experiencias $^{1}$ a escala regional que pretendían arraigar espacios de debate referidos a los temas de la ciudad, el urbanismo y la arquitectura, estas actividades no logran conformar lo que Edward Shils (1970) identifica como del proceso de institucionalización, entendido como la existencia de una relativa y densa interacción de personas que conducen una actividad dentro de un convenio social que tiene límites, resistencias y nominaciones. A su vez, lo define a partir de la consolidación y diagramación de la enseñanza y la investigación en condiciones de organización regulada, programada y sistemáticamente administrada y, en el último lugar, identifica que el proceso se da a partir de la existencia de posibilidades de publicación y difusión de los resultados de la actividad y la accesibilidad de los mismos al público. Estas condiciones, asociadas a la idea de tradición ${ }^{2}$ común, permiten definir los indicadores para comprender los procesos de institucionalización de una disciplina y los niveles de transformación en

1 Entre las experiencias podemos mencionar los Congresos Panamericanos de Arquitectos (1920/1927), los Congresos Panamericanos de Municipio (1938/1941) o los Congresos Panamericano de la Vivienda Popular (1939).

$2 \quad$ Edward Shils sostiene que la tradición es un agregado heterogéneo de tópicos relacionados entre sí por un nombre común, por técnicas más o menos comunes, por una comunidad de palabras claves y concepciones, por un agregado mayor de ideas y por un conjunto de esquemas conceptuales e interpretativos. 
el tiempo.

SIAP se imagina como un espacio de intercambio de experiencias a escala regional con el fin último de implementar directrices científicas y tecnológicas tendientes al desarrollo de los países latinoamericanos. Este objetivo implica la delineación o re-adaptación de los principios de la planificación democrática participativa en clave "latinoamericana", identificando la apropiación ${ }^{3}$ de un modelo que en principio puede pensarse exógeno, pero que en realidad ya está parcialmente "transformado" por el aparato conceptual de las organizaciones supranacionales actuantes en América Latina, particularmente las innovaciones que darán forma al repertorio teórico de la CEPAL, como son las relaciones "centro-periferia", el desequilibrio estructural y la planificación del desarrollo en América Latina e integración regional, por nombrar sólo algunas.

Los objetivos de la Sociedad se organizan a partir de tres acciones interconectadas. Por un lado el conocimiento de las realidades locales que implica no sólo el levantamiento de datos, sino principalmente la construcción de un andamiaje teórico-metodológico adaptado a los cambiantes factores causales (políticos, sociales y económicos) que caracterizan la realidad latinoamericana. En segundo término, la difusión del mensaje planificador, tanto en términos académicos como en las esferas de toma de decisiones y, por último, la promoción e institucionalización de programas académicos a nivel de maestría y doctorado que permitan formar "expertos" capaces de integrarse a las oficinas técnicas estatales a escala nacional, provincial y municipal.

La renovación disciplinar a escala latinoamericana, donde la variable física/territorial es una más junto a la política, la economía y lo social, en los intentos por lograr el tan deseado equilibrio es acompañada por la generación de sociedades o comisiones ${ }^{4}$ de trabajo que operan en un sistema interconectado a nivel regional, interviniendo en la organización de reuniones, seminarios de formación, programas académicos, así como también en la publicación de libros y revistas que van a convertirse en los espacios de difusión del

$3 \quad$ Entendemos a la apropiación como un acto de recepción activa, capaz de transformar las ideas de "origen" a partir del matiz de la propia realidad, generando nuevas recombinatorias que la convierten en propias.

$4 \quad$ En el año 1966 se consolida la Comisión de Desarrollo Urbano y Regional (CDUyR) en el marco del Consejo Latinoamericano de Ciencias Sociales (CLACSO). La particularidad de la CDUyR radica en el funcionamiento corporativo a partir de la asociación de los centros nacionales. Para ampliar sobre el funcionamiento de la comisión ver Jajamovich Guillermo. (2013) Itinerarios de la Comisión de Desarrollo urbano y regional de CLACSO entre 1967 y 1973: entre la técnica y la política, las ciencias sociales y la planificación urbana y regional. Congreso XXIX ALAS, Santiago de Chile. mensaje planificador en la región.

Si entendemos a la SIAP como una nueva forma de institucionalización de la disciplina, en su programa de trabajo se presentan los intentos por instaurarse en la región como el espacio condensador de la planificación a partir de "la invitación a las sociedades locales de planificación de Estados Unidos y de los diferentes países de América Latina a que se afiliaran a SIAP para coordinar el esfuerzo de estimular el desarrollo, el uso y los conocimientos de la planificación integral" (Camacho, 2007:268).

De esta forma, la sociedad actúa como una institución que aglutina los centros, las instituciones y los actores del continente, convirtiéndose en un espacio de consolidación del ideal planificador desarrollista del período y, por lo tanto, como un espacio en el que los grupos profesionales van a controlar los criterios de su propio saber, delineando los límites y construyendo los espacios del saber técnico del experto.

Mariano Plotkin y Federico Neiburg, definen al experto como "los técnicos, los especialistas que trabajan en el Estado, y más recientemente para las ONGs, y los organismos internacionales. "(...) la figura del experto evoca especialización y entrenamiento académico, actuando en nombre de la técnica y de la ciencia, reclamando hacer de la neutralidad axiológica la base para la búsqueda del bien común..."(2004:15). Su actividad se sustenta en principios científicos, en el uso de la técnica para la "construcción de discursos y narrativas con pretensión de validez" (Morresi y Vommaro, 2011:13). Su consolidación en el campo de la planificación regional y urbana se produce a partir de la articulación entre la renovación de los postulados teóricos metodológicos y la respectiva ampliación de la "oferta de saber ligada a la definición de problemas a los que se ofrecen soluciones adaptadas" (Morresi y Vommaro, 2011:15) y la intervención en la región de nuevas estructuras institucionales a diferentes escalas que inauguran un modelo alternativo de prestación de servicios respecto a la del profesional liberal.

\section{SIAP y su proyecto editorial}

A partir del año 1966 SIAP inicia su programa editorial promovido por la presidencia del planificador argentino Jorge Enrique Hardoy. Bajo la denominación de Ediciones SIAP, el programa se articuló a partir de cuatro líneas diferenciadas: la publicación de libros, la redacción de la Revista de la Sociedad Interamericana de Planificación, la publicación del Boletín Correo Informativo SIAP y, por último, la publicación de las actas de los congresos y seminarios (Camacho, 2007: 268).

Cabe destacar que cada uno de los programas de Ediciones SIAP contaba con sedes diferenciadas. En el caso de los Libros Ediciones SIAP, cuya publicación se inicia en el año 1970, la sede 
Figura 1: Distribución de las sedes de Ediciones SIAP

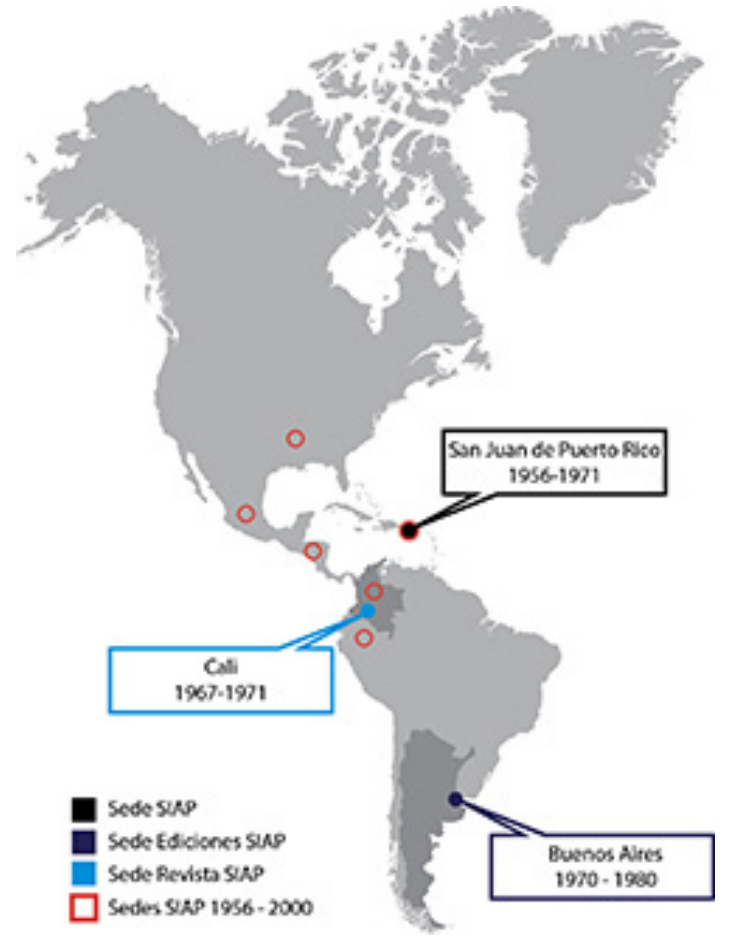

Fuente: elaboración propia

fue la ciudad de Buenos Aires ${ }^{5}$, en línea con el crecimiento a escala regional de la industria editorial argentina que demostraba su capacidad de distribución a América Latina y el resto del mundo a partir de un convenio de exclusividad con la casa editorial Ediciones Nueva Visión S.A., fundada por miembros de oam a mediados de la década del cincuenta.

La Revista SIAP y los Boletines tienen su sede en la ciudad de Cali, vinculada al Departamento de Administración Pública y Planificación, División de Ciencias Sociales y Económicas de la Universidad del Valle. Esta situación se mantiene hasta 1971, donde la sede de la revista pasa a la ciudad de Bogotá.

La particularidad de SIAP en este período radica en la descentralización de su estructura institucional, ya que la Secretaría General se ubicaba en la ciudad de San Juan de Puerto Rico, la edición de libros en Buenos Aires y de la revista en Cali, sumado a la rotación de los congresos y actividades, y a la conformación plural de los equipos directivos y técnicos que guiaron los pasos de la institución en sus diferentes etapas.

Esta búsqueda de "equilibrio" tiene como objetivo, como afirma Camacho, "lograr un mayor desarrollo en los países del continente americano

$5 \quad$ Entre 1970 y 1980 el programa editorial funciona en Buenos Aires. A partir de 1980 la sede se traslada a la ciudad de México, realizando las actividades directamente desde la Secretaría General de SIAP. con una más equitativa distribución del mismo entre su población y su territorio, mediante los principios y técnicas de la planificación democrática y participativa" (2007: 269). (Figura 1)

\section{SIAP la revista}

Con la creación de la Revista de la Sociedad Interamericana de Planificación ${ }^{6}$ se consolida un proyecto editorial que refuerza aún más el rol de SIAP a escala regional. Esta situación le asigna a la publicación un rol relevante como espacio de sintetización y difusión de los principios y objetivos de la Sociedad.

En la elección del primer período de la revista, correspondientes a las publicaciones producidas entre los años 1967 y 1970 (15 números), se mantienen ciertas condiciones que permiten la comprensión del rol de la misma en el contexto disciplinar latinoamericano. En primer lugar, la estructura institucional que le da forma se mantiene estable, conservando la dupla de dirección de SIAP y la revista en las manos de Jorge Enrique Hardoy y Pablo Pedro Morcillo respectivamente. En segundo término, este período funciona como un prisma que permite hacer visibles los modos en que la revista opera en la construcción y la consolidación del discurso de la planificación regional y urbana en clave cepalina en América Latina.

Además del director, el equipo editorial de la revista ${ }^{7}$ se complementa con un comité editorial y corresponsales editoriales nacionales, sumado a la secretaría administrativa y la secretaria editorial. Si bien los actores intervinientes van a variar en el tiempo, resulta significativa la composición del primer comité, integrado por académicos norteamericanos y latinoamericanos que mantenían en su mayoría vínculos con organismos supranacionales como Naciones Unidas, el Banco Interamericano de Desarrollo, CLACSO y la Fundación Ford.

En la editorial del primer número se expone la importancia del proyecto para SIAP, identificándolo como "uno de sus proyectos más importantes y por todos los socios largamente deseado" (Morcillo, 1967:2), intentando convertirse en un espacio de intercambio de ideas y experiencias que permita a los planificadores del continente una constante actualización de los temas y enfoques que van delineando la disciplina.

\footnotetext{
$6 \quad$ En total Revista SIAP publica 120 números, a razón de 4 por año.

$7 \quad$ En los primeros catorce (14) números el equipo editor se completaba con la Secretaria Editorial, Judith de Campos; la Secretaría Administrativa, Rosalba Restrepo y el Comité Editorial Asesor integrado por John Friedmann, Aldo Ferrer, Ralph Gakenheimer, Helio Jaguaribe, Ricardo Jordán, Alberto Mendoza, Eduardo Montoulieu y Luis Vera; sumado a un conjunto de corresponsales nacionales.
} 
Su organización se divide en cuatro secciones (artículos, apuntes y notas, difusión y bibliografías), sumado al texto que encabeza cada número y que, a cargo del editor, organiza los diferentes núcleos temáticos ${ }^{8}$.

\section{Revista SIAP. Los temas centra- les}

La selección del apartado "Artículos" para el análisis, se presenta como un camino para la identificación de los temas teóricos y metodológicos que delinean el proyecto editorial de la revista. Cuatro son los números publicados durante el primer año. Los mismos albergan diecinueve artículos con temáticas diferenciadas.

Los temas propuestos se organizan a partir de tres tipos de enfoques ${ }^{9}$. Dentro del primer grupo, que podríamos denominar como doctrinario, se encuentra la discusión sobre la definición de la planificación, sus variaciones y matrices de referencia ${ }^{10}$. En este sentido, es posible advertir los posicionamientos críticos a la interpretación "abstracta" de la planificación, sumado a la centralidad de los abordajes enfocados en los aspectos económicos de la planificación en detrimento de enfoques sociales o políticos. La propia delimitación de la práctica profesional y sus agencias se enmarca dentro de esta discusión permitiendo una lectura que avanza en los vínculos entre Estado, organismos de financiación y asesoramiento externo (supranacionales o regionales) y la matriz teórica/metodológica.

La formación profesional va a guiar el segundo grupo de textos ${ }^{11}$, reconociendo no sólo los

$8 \quad$ En la sección de artículos se presentan los textos centrales de la revista, definiendo las líneas temáticas de cada número. En apuntes, se presentan escritos de opinión o avances de investigación tanto referidos a los temas e ideas, como así también la discusión de casos y trabajos empíricos. En el apartado de difusión se reúne la información referida a eventos, cursos, becas de formación y trabajos de asistencia en la región, conformándose como una agenda regional que le permitan al lector permanecer informados y actualizado sobre las últimas noticias. El último apartado se orienta a la difusión de bibliografías, libros, revistas y folletos, identificando la centralidad de la publicación de experiencias de planes y proyectos de desarrollo.

9 Cabe destacar que los artículos del número cuatro exponen una selección de las ponencias del $1^{\circ}$ Seminario Interamericano sobre la definición de regiones para la planificación del desarrollo, realizado en Canadá en el año 1967.

10 Dentro de este grupo podemos mencionar a "La planificación como innovación" de John Friedmann; "Gobierno, planificación y desarrollo económico" de Rafael Picó; "Desarrollo planificado y transformaciones socio institucionales" de Julio Cesar Funes y Rosario Scarpati; "América Latina ante las sociedades alienadas" de Alberto Mendoza; "Obstáculos al desarrollo: una clasificación y quasi eliminación" de Albert Hirschmann.

$11 \quad$ Entre este grupo de textos se destaca " La enseñanza en el campo del desarrollo urbano regional en diferentes modelos existentes en América Latina sino, principalmente, buscando una discusión que ponga en relación las estructuras académicas con las necesidades reales de la región.

En el tercer grupo, se presentan una serie de estudios de casos que proponen la revisión entre teoría y práctica. Los ejemplos trabajados: Puerto Rico, Venezuela y el nordeste de Brasil, son sinónimos de las primeras experiencias planificadoras bajo el lenguaje desarrollista, actuando como faro para el resto del continente tanto a nivel de aciertos como de fracasos. Si la revista funciona como el espacio de difusión de SIAP, los temas presentados van a identificar las discusiones por la definición disciplinar y los intentos por monopolizar un saber que, en base a una interpretación de la planificación como un concepto abarcante de aspectos y factores económicos, sociales, físicos y políticos, asume a la interdisciplina como un elemento determinante, condición que requiere una nueva estructura de enseñanza que permita formar profesionales capaces de adaptarse a las nuevas exigencias regionales y, por lo tanto, construir un monopolio respecto de su propia práctica.

En el segundo año, entre septiembre de 1968 y el mismo mes de $1969^{12}$, los temas de los artículos se van a organizar en torno a dos grandes ejes, pero con ciertas variaciones respecto del año anterior: en primer lugar, los estudios de casos van a adquirir un mayor protagonismo, en parte esta condición se presenta por el significativo avance de las investigaciones en la región y la conformación de centros e instituciones que indagan sobre el estado del desarrollo urbano en los países de América Latina. Sin embargo, estos escritos no se proponen como mera recopilación y diagnóstico de los casos nacionales, sino que van a conformar un corpus de experiencias que proporcionan una plataforma para la discusión de uno de los temas centrales que atraviesa al período: los alcances reales de la reforma urbana y la reforma agraria. A partir del número $9 / 10$, los casos dejan de referirse a las experiencias nacionales, identificando un giro que vincula temas y territorios específicos, como por ejemplo los sistemas de ciudades en Venezuela, la organización institucional del área metropolitana de Buenos Aires o bien, los modelos administrativos de la tierra en Puerto Rico. Esta condición supone un abandono de la macro escala y la respectiva adaptación de ciertos temas a los contextos latinoamericanos, estableciendo la creciente necesidad de producir un andamiaje teórico y conceptual que permita redefinir ciertas nociones y posicionamientos que

Chile. Nuevo Enfoque" de Guillermo Geisse y "Propuesta para la reestructuración de la PUC de Rio de Janeiro" de Rudolph Atcon

$12 \quad$ Este período lo componen los números 5 y 6 , $7,8,9$ y 10 , y el número 11 . 
identifican al desarrollo como el fin último a alcanzar por los países "atrasados".

Una particularidad de este período es la desaparición entre las páginas de la discusión sobre la enseñanza de la planificación, situación que se corresponde con la creciente estabilidad y consolidación de los centros regionales, sumado a la generación de programas en el marco de las estructuras universitarias que, más allá de ciertas variaciones, contribuyen a la formación de los "expertos" en el campo de la planificación regional y urbana latinoamericana.

Los encuadres teóricos y metodológicos de los artículos en estos números demuestran el proceso de definición de los alcances de la disciplina, reconociendo por un lado los diagnósticos demográfico, económicos y físicos de la región y, por el otro, la noción de la planificación como método propositivo que permite delinear aspectos naturales, sociales, económicos y políticos en función de una idea de equilibrio.

Siendo 1969 el año de cierre de la denominada "década del progreso", comienza a advertirse en los artículos un progresivo corrimiento a posiciones más críticas respecto de las implicancias del desarrollo, identificando la crisis del sistema estructuralista del crecimiento económico de la región y la imposibilidad de mantener un equilibrio entre crecimiento y desarrollo demográfico, situación que va a cristalizar la crisis de las metas del desarrollo propuestas para la región.

Este incipiente corrimiento de las implicancias del desarrollo se va a acrecentar en los números posteriores, ya que no sólo se proponen variaciones que representan una revisión de las ideas de la planificación nacional y regional, sino también una nueva mirada a las instituciones supranacionales que actúan en la región, reconociendo a la "ayuda" externa como un valor en un momento determinado para potenciar el desarrollo pero que, con el paso del tiempo, no ha hecho más que aumentar la dependencia de la región ante lo inacabado del "proyecto".

Esta crisis se muestra en una serie de artícu$\operatorname{los}^{13}$ que vuelven a ubicar a América Latina en el centro de la discusión, introduciendo un giro en el discurso que abandona paulatinamente la visión del desarrollo reformista, en los términos propuestos por Gorelik (2014), e introducen la noción de dependencia como categoría coyuntural revisitando las estructuras políticas y sociales, además de lo económico, como clave para la planificación de las transformaciones en el con-

$13 \quad$ Entre los artículos de este grupo podemos destacar: "La regionalización de las políticas de desarrollo en América Latina" de Eduardo Neira Alva; "El espacio físico en la política de desarrollo" de Carlos Matus Romo; "El concepto de desarrollo polarizado en planeación regional: un enfoque sociológico" de Torcuato Di Tella (h)
Figura 2: Distribución de los artículos por ejes temáticos

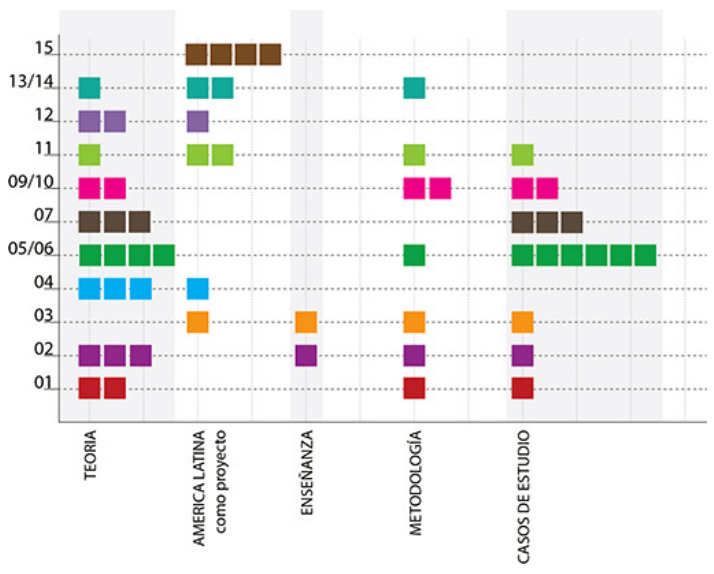

Fuente: Elaboración propia

tinente.

El último número de esta serie, de septiembre de 1970, cristaliza este proceso a partir de una revisión de los aspectos económicos del desarrollo, sociales, geográficos y políticos, fortaleciendo una revisión conceptual, no centrada ahora en la delimitación de la planificación como método y en el territorio latinoamericano como laboratorio, sino de las condiciones estructurales de América Latina y las posibles líneas de acción desde un marco teórico-conceptual producido por actores e instituciones locales ${ }^{14}$.

Una particularidad de la diagramación de la revista la constituye la progresiva eliminación de los resúmenes en idioma inglés. Si en los primeros números cada artículo se acompañaba con un asbtract, desde el número siete al once estos sólo acompañan ciertos textos y no se ubican en la primera página del escrito. Desde diciembre de 1969, se produce la eliminación definitiva del inglés en las páginas de la revista, condición que en principio se plantea como anecdótica, pero que en realidad refuerza el cambio de orientación de la revista en su afán por consolidar una mirada local desde y para América Latina que implica la utilización del español como "el" idioma de SIAP. (Figura 2)

\section{Revista SIAP. Los autores}

Si bien el análisis de los temas publicados en la sección de artículos, permite trazar un perfil de los modos en que se aborda el desarrollo la disciplina en el período. El estudio del conjunto de autores participantes proporciona un mapa que

$14 \quad$ Entre los artículos de este grupo podemos destacar: "Planteamientos sobre el desarrollo económico de América Latina" de Norberto González; "Tendencias del desarrollo socio-económico de América Latina" de Fernando Enrique Cardoso; "Aspectos Geográficos de la Planificación en América Latina" de Harold Wood o "Aspectos Geográficos de la Planificación en América Latina" de Marcos Kaplan. 
hace visible los cruces entre actores, centros de investigación y organismos en las múltiples escalas que actuaron en América Latina.

En cada artículo, la revista presenta una descripción biográfica breve de los autores. En los primeros once números, la misma mantiene un promedio de siete líneas, identificando las actividades más representativas de la trayectoria, su formación de grado y posgrado, sumado al recorrido institucional. A partir del número doce, la longitud de los datos biográficos se reduce notablemente identificando, mayoritariamente, el espacio institucional del autor al momento de la redacción del escrito.

Un escenario posible para explicar la reducción de información se define a partir del reconocimiento de la necesidad conjunta de legitimizar el saber experto y al propio experto en un campo en proceso de consolidación que en los primeros números se está tratando de fortalecer. Esta situación no sólo le otorga importancia al texto publicado, sino también a quién y desde que espacio político/técnico o académico está operando, valorando las diferentes trayectorias de los autores.

Si en el primer año los intentos están centrados en la consolidación de la disciplina, en "la aplicación de los principios, métodos y técnicas..." (Morcillo, 1967, p.2) y en la conformación de un saber "propio" para los países latinoamericanos, resulta significativo el relevamiento de actores, siendo que de los veinte, once son latinoamericanos, cuatro europeos, dos norteamericanos y dos canadienses. Si a esta cuantificación un tanto "engañosa", le cruzamos la formación de posgrado, vamos a advertir que entre los latinoamericanos seis por lo menos realizan una maestría o un doctorado en los Estados Unidos. Esta situación implica uno de los factores centrales para la comprensión de la consolidación de los principios de la planificación democrática norteamericana en clave desarrollista en América Latina, sumado a la importancia otorgada a los espacios universitarios y, particularmente a la formación de posgrado en el extranjero, como lugares de consolidación del saber experto.

El segundo enfoque para analizar el mapa de autores, radica en la revisión de su filiación institucional, condición que revela que la totalidad de los latinoamericanos se encuentran vinculados a alguno de los centros creados en la región en la década del sesenta (CENDES, CEUR, CIDU). También cabe destacar que dentro de estos autores se encuentran personalidades relevantes de SIAP como el caso de Picó que fue el primer presidente de la misma o Hardoy que era el presidente durante la publicación de la revista. A su vez, de la totalidad de los autores no latinoamericanos la mitad son asesores de un organismo supranacional, principalmente ONU y CEPAL, o bien asesores financiados por la Fundación Ford.
Figura 3: Mapa de autores Revista SIAP 1967-1968
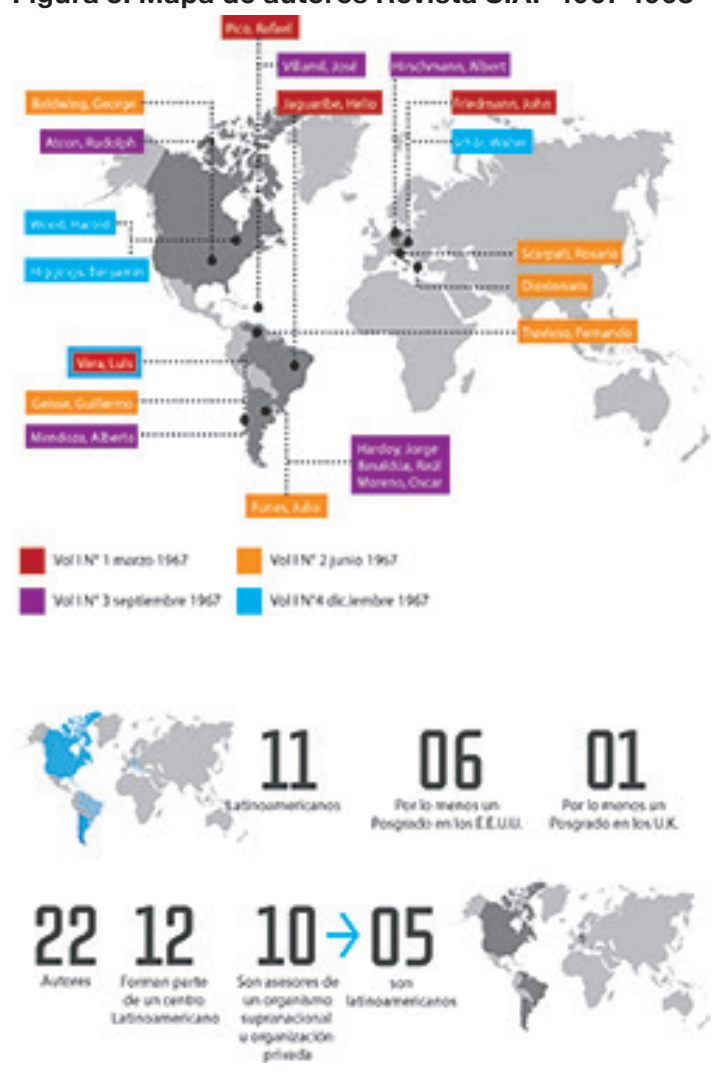

Fuente: Elaboración propia

Entre los latinoamericanos que cumplen este rol encontramos al puertorriqueño Rafael Picó, al chileno Luis Vera y al argentino Julio César Funes.

Con la ampliación del desarrollo de casos el mapa de actores publicados en la revista se transforma. La presencia latinoamericana aumenta significativamente llegando a un $75 \%$ sobre un total de cuarenta y uno, mientras que el resto se completa con una marcada presencia norteamericana.

A pesar de que Chile se consolida en la región como un espacio de concentración de organismos y centros de la planificación, la presencia en la revista de autores chilenos (3) no concuerda con esta centralidad, mientras que entre Argentina (9) y Colombia (5) concentran el $50 \%$ de los autores ${ }^{15}$.

En materia de formación de posgrado, la tendencia del período anterior se mantiene, identificando que más del $65 \%$ cuenta con una alguno de los dos niveles, marcando una predominancia de las universidades norteamericanas (15) por sobre las europeas (5). Harvard y Penn lideran la elección de los latinoamericanos por las casas de altos estudios norteamericanas, seguidas por Columbia, Berkeley, Yale y el MIT.

15 El resto se distribuye: México, 1; Brasil, 2; 2 
Con respecto a la filiación institucional, en el segundo período podemos observar el incremento de actores latinoamericanos actuando como asesores de organismos supranacionales como la ONU, la OEA o el BID, como el caso del peruano Neira Alva, del cubano Mountoulieu o el chileno Matus Romo. Aparecen también los espacios para asesoramiento a los gobiernos nacionales o municipales como el caso del venezolano Funes que presta servicios de asesoría a su país o el cubano Castañada que asesora a la Junta de Planificación de Puerto Rico. A escala municipal, el abogado argentino Mouchet presta su servicio experto asesorando al intendente de la ciudad de Buenos Aires, a partir de su rol como director del grupo de estudio del área metropolitana de la ciudad en el marco del programa internacional Bureau of Municipal Research de Canadá.

Entre los autores norteamericanos, la tendencia del periodo anterior se mantiene, produciéndose una división entre gobiernos locales como el caso del sociólogo Smith a Brasil, las asesorías a organismos supranacionales como el caso de Wood, o bien el trabajo para fundaciones privadas como el caso de Friedmann y Gakenheimer para la Fundación Ford en Chile.

Nuevamente, los centros de la región cuentan con representantes entre las filas de la revista, reconociendo la ampliación institucional y geográfica de los mismos como el caso del Centro de Estudios Económicos y Demográficos (CEED) en México y el Centro Interamericano de Vivienda y Planeamiento (CINVA) en Colombia, sumado a la creciente centralidad que adquiere el organismo de la CEPAL el Instituto Latinoamericano y del Caribe de Planificación Económica y Social (ILPES) en Chile.

A partir de este análisis, se identifica que en el segundo y tercer año de la revista el mapa de actores, coincidente con el tratamiento de los temas, tiene una predominante presencia latinoamericana, que posibilita identificar la maduración y estabilización de la disciplina en la región, reconociendo como factores centrales la filiación institucional de los actores que permiten advertir sobre la ampliación de la red de centros y espacios de formación e investigación referidos a la planificación regional y urbana. Un segundo factor lo demuestra el cambio en las asesorías, que ahora se reparten entre latinoamericanos y norteamericanos (mayoritariamente), identificando el reconocimiento por parte de los gobiernos locales y las organizaciones supranacionales de un saber experto local, legitimizado por su formación en el extranjero pero que, de igual modo consolida un espacio alternativo de trabajo para los planificadores latinoamericanos. (Figura 4).

\section{Figura 4 Mapa de autores Revista SIAP 1968-1970}

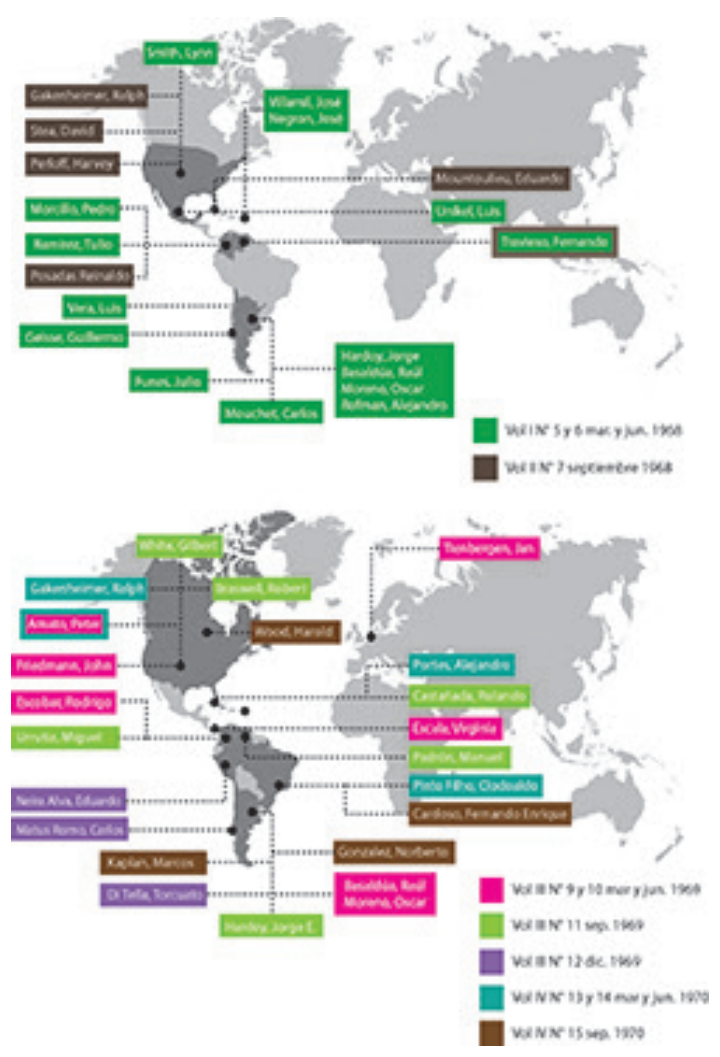

Fuente: Elaboración propia.

\section{El rol de la Revista SIAP en la consolidación de la disciplina en América Latina}

La lectura del primer período de la Revista SIAP posibilita una mirada de los intentos de consolidación de un saber específico referido a la planificación regional y urbana desde el órgano de difusión de la Sociedad. Esta condición no sólo plantea una redefinición de los principios de la planificación en clave Latinoamericana a partir de la delineación de una nueva matriz teórica-metodológica, sino también la consolidación de un espacio de intercambio y diálogo entre actores e instituciones locales, regionales y supranacionales que desde múltiples ángulos van a construir una expertise que se define por el proceso importación/adaptación, pero que con el tiempo va construyendo saberes y redes que sostienen el discurso experto de la planificación desarrollista en clave cepalina en América Latina.

El análisis de los artículos publicados en la revista posibilitó comprender el progresivo giro de posiciones tendientes a definir los contornos disciplinares, sus temas y actores, para dar paso a una preponderancia de estudios de casos, donde prevalecen los enfoques nacionales y las miradas regionales que permite el itinerario temático que va a atravesar a la región, desde un posicionamiento del subdesarrollo como una etapa posible 
de ser revertida a críticas sustentadas en la condición estructural de las bases del atraso, sumado a la creciente dependencia a las instituciones supranacionales que en definitiva no hacen más que aumentar el propio mal que habían diagnosticado en los inicios de la década del sesenta: la dependencia económica de la región.

Este período de la Revista SIAP expone este paulatino proceso, identificando a su vez las redes institucionales que van a protagonizar el debate disciplinar no sólo a escala de sociedades nacionales de planificación, centros de investigación o espacios universitarios, sino también a partir de la acción de organismos supranacionales y fundaciones privadas que van a "asistir" a la región tanto económicamente como técnicamente.

En esta línea, en los sucesivos números resulta posible identificar el cambio de autores, que en un inicio cuenta con una mayor presencia de actores internacionales pero que, a medida que avanza la publicación, va a incorporar progresivamente actores del medio latinoamericano, muchos de los cuales participan activamente del desarrollo de la disciplina en la región no sólo desde el ámbito de formación e investigación, sino también desde la asistencia técnica a instituciones públicas de diferentes escalas nacionales, o bien, en las organizaciones supranacionales que operan en la región.

Estas condiciones permiten identificar a la Sociedad como una institución central del proceso de consolidación de la disciplina regional y urbana en Latinoamérica, sumado al reconocimiento de la Revista SIAP como un espacio de afirmación del saber experto que va haciendo visible, a través de sus escritos, los vaivenes del proceso de transformación de los temas y problemas que permiten redefinir los posicionamientos disciplinares en el período. 


\section{Bibliografía}

Almandoz, A. (2010). From urban to regional planning in Latin America, 1920-50, Perspectives 25, (1), enero.

Almandoz, A. (2006), Urban planning and historiography in Latin America, Progress in planning (65) Camacho, L. (2007). Sociedad Interamericana de Planificación, SIAP 50 años Vida institucional y programática, Revista Bitácora Urbano Territorial, enero-diciembre.

Gorelik, A. (2014). Miradas cruzadas. El viaje latinoamericano del planning norteamericano, Bifurcaciones, (18), septiembre/noviembre. Disponible en. http://www.bifurcaciones.cl/2014/12/gorelik/

Jajamovich, G. (2013). Itinerarios de la Comisión de Desarrollo urbano y regional de CLACSO entre 1967 y 1973: entre la técnica y la política, las ciencias sociales y la planificación urbana y regional. Ponencia presentada en el Congreso XXIX ALAS, Santiago de Chile.

Morresi, S. y Vommaro G. (Comp.) (2011). Saber lo que se hace. Expertos y política en Argentina. Buenos Aires: Prometeo Libros

Neiburg, F. y Plotkin, M. (Comp.) (2004), Intelectuales y expertos. La constitución del conocimiento social en la Argentina. Buenos Aires: Paidós.

Sarfatti Larson, M. (1988). El poder de los expertos. Ciencia y educación de masas como fundamento de una ideología, Revista Educación, (285), Madrid, enero-abril.

Shils, E. (1970)., Tradition, ecology, and institution in the history of sociology en The calling of sociology and other Essays in the pursuit of learning. Selected papers III, University of Chicago, Chicago.

\section{Fuentes}

Revista SIAP Vol I No 1 marzo 1967

Revista SIAP Vol I N² junio 1967

Revista SIAP Vol I N³ septiembre 1967

Revista SIAP Vol I N4 diciembre 1967

Revista SIAP Vol I N5 y 6 marzo y junio 1968

Revista SIAP Vol II N 7 septiembre 1968

Revista SIAP Vol III Nº y 10 marzo y junio 1969

Revista SIAP Vol III N 11 septiembre 1969

Revista SIAP Vol III N 12 diciembre 1969

Revista SIAP Vol IV N 13 y 14 marzo y junio1970

Revista SIAP Vol IV N 15 septiembre 1970 